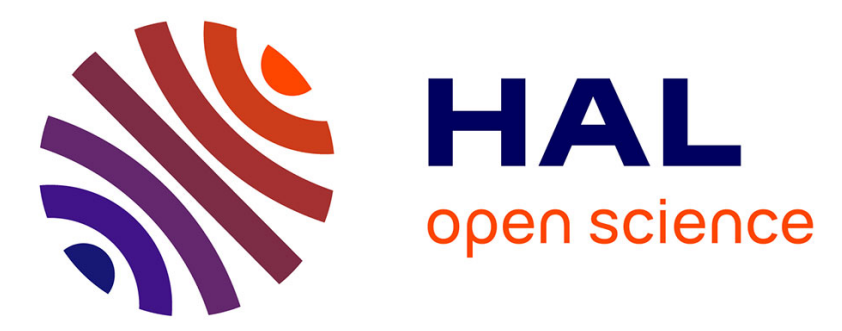

\title{
In Situ Transmission X-ray Micro-Diffraction from Thin Metal Films Electrodeposited in Microfluidic Channels
}

T. Wiegmann, J. Drnec, F. Reikowski, J. Stettner, F. Maroun, O. Magnussen

\section{To cite this version:}

T. Wiegmann, J. Drnec, F. Reikowski, J. Stettner, F. Maroun, et al.. In Situ Transmission X-ray Micro-Diffraction from Thin Metal Films Electrodeposited in Microfluidic Channels. Journal of The Electrochemical Society, 2020, 167 (11), pp.112505. 10.1149/1945-7111/aba077 . hal-03080001

\section{HAL Id: hal-03080001 https://hal.science/hal-03080001}

Submitted on 4 Jan 2021

HAL is a multi-disciplinary open access archive for the deposit and dissemination of scientific research documents, whether they are published or not. The documents may come from teaching and research institutions in France or abroad, or from public or private research centers.
L'archive ouverte pluridisciplinaire HAL, est destinée au dépôt et à la diffusion de documents scientifiques de niveau recherche, publiés ou non, émanant des établissements d'enseignement et de recherche français ou étrangers, des laboratoires publics ou privés. 


\title{
In situ Transmission X-ray Micro-Diffraction from Thin Metal Films Electrodeposited in Microfluidic Channels
}

T. Wiegmann ${ }^{1,2}$, J. Drnec ${ }^{2,}{ }^{*}$, F. Reikowski ${ }^{1}$, J. Stettner ${ }^{1}$, F. Maroun ${ }^{3,}{ }^{*}$, O. M. Magnussen ${ }^{1,{ }^{*}}$

${ }^{1}$ Institute of Experimental and Applied Physics, Kiel University, 24098 Kiel, Germany

2 Experimental division, ESRF, 71 Avenue des Martyrs, 38000 Grenoble, France

${ }^{3}$ Laboratoire de Physique de la Matière Condensée, CNRS, IP Paris, 91128 Palaiseau, France

\begin{abstract}
High-energy X-ray surface diffraction in transmission geometry is combined with a microfluidic thin layer flow cell for in situ studies of the local structure of electrodeposited epitaxial films. The capabilities of this approach are illustrated using Bi films on $\mathrm{Au}(100)$ single crystals as an example. We demonstrate that the local film thickness, the strain, and the orientation of the deposits' crystallites can be mapped with a spatial resolution of a few micrometers. The high heterogeneity of the Bi films provides deposits with a wide range of structural properties, allowing to establish correlations between the different parameters as a function of the local thickness.
\end{abstract}




\section{Introduction}

The interface between a solid material and a liquid or a high-pressure gas phase is often subject to complex and heterogeneous structure changes, and understanding such processes from an atomistic perspective is of high importance in electrochemistry, catalysis, lithography and many other areas of the material sciences. Synchrotron-based surface X-ray diffraction (SXRD) is one of the few methods available for in situ structural studies of condensed matter interfaces. Especially for the study of single crystal samples under ambient conditions, SXRD's ability to resolve the full atomic structure with picometer precision makes it the technique of choice in many cases, including studies in electrochemical environments.[1-4]

SXRD experiments are conventionally performed in grazing incidence geometry (GID), where the X-ray beam impinges at close to the critical angle, increasing the surface sensitivity. The resulting diffraction signal consists of Bragg diffraction spots originating from the bulk lattice as well as crystal truncation rods (CTRs, a nonzero intensity variation along the out-of-plane direction between Bragg peaks) originating from the interface. Their shape and position can be used to determine the structure of the substrate surface, surface reconstructions, and adsorbed monolayers or thin films.[5-14]

When used at typical SXRD energies (15 - $25 \mathrm{keV}$ ), the GID geometry usually requires rotating both the sample and the detector to obtain a full diffraction profile of CTRs, which may be time-consuming. Gustafson et al. showed that using high photon energies ( $85 \mathrm{keV}$ ) and large $2 \mathrm{D}$ detectors enables faster measurements, as the reduced curvature of the Ewald sphere at high energy allows a much greater portion of the relevant CTR to be visible on the detector in one acquisition.[15]

We recently demonstrated a novel technique termed Transmission Surface Diffraction (TSD), where a high-energy $\mathrm{X}$-ray beam $(70 \mathrm{keV}$ ) impinges the sample at near vertical incidence (i.e. close to the surface normal) and then passes through the bulk of the sample and the interface region (Fig. 1).[16] In this configuration, the Ewald sphere lies almost parallel to the interface and a direct visualisation of the in-plane reciprocal space geometry is projected onto the detector, with diffraction peaks appearing at intersections between the Ewald sphere and CTRs or adsorbate diffraction rods. TSD therefore produces images similar to low-energy electron diffraction (LEED) and reveals the orientation and symmetry of the interface and all reconstructed and adsorbed phases in a single acquisition.

Compared with SXRD under grazing incidence, the main benefit offered by TSD is the small beam footprint on the sample. While in GID this footprint is on the order of several millimetres even for small X-ray beams, in TSD it is only limited by the size of the beam itself, typically less than a few micrometres. A wide range of real-world interface systems in technological devices or at natural materials such as oxides, perovskites and 2D materials exhibits heterogeneities at this scale. Combining its advantages of small beam footprint, single-shot, LEED-like structure identification, capability for measuring in various environments, and low sensitivity to alignment errors, TSD is a powerful tool for the study of such systems. In proof-of-principle experiments, we demonstrated that TSD is surfacesensitive down to single and even partial monolayers for heavy scatterers ( $\mathrm{Au}, \mathrm{Bi})$.[16] 
Given the advantages described above, TSD is an ideal tool to investigate electrochemical systems in thin layer flow cells or microfluidic cells. This is of high relevance for electrodeposition-based fabrication, manipulation and degradation of electronic systems [17, 18] or for electroanalytical chemistry and chemical sensing.[19] In these applied systems, finely tuned control of the electrolyte flow field produces deposition heterogeneities that are often on the micrometre scale and therefore inaccessible to GID. In contrast, the combination of TSD with a microfluidic device allows in situ mapping of in-plane structure parameters over the electrode surface or operando monitoring of dynamic electrochemical processes at specific sample positions. Such spatially resolved information is very difficult to obtain with any other analytical technique, making this combination a unique tool in a general sense. In addition, TSD offers other methodological advantages: First, it can probe picometer-scale parameters such as surface strain or texture, which are not accessible to microscopy-based in situ techniques (e.g. EC-STM, EC-AFM). Second, contrary to ex situ techniques, such as electron microscopy or diffraction, it does not require a sample transfer and therefore can be easily applied to samples that are unstable in air. In general, it can access information from deeply buried interfaces, which is not feasible for most techniques that are not based on hard $\mathrm{X}$-rays.

In the present work, we use the combination of TSD and thin layer microfluidic cell to study the electrodeposition of $\mathrm{Bi}$ on $\mathrm{Au}(100)$ electrodes. In particular, we show how this can be used to characterize the spatial distribution of structural parameters such as film thickness, strain or orientation disorder over the electrode surface and how these heterogeneities can be related to the local thickness of the electrodeposit. To demonstrate the capabilities of this new in situ characterization technique, we employ diffusion-limited deposition conditions which lead to heterogeneous deposits.

\section{Experimental}

\subsection{Diffraction setup and electrochemical cell}

Experiments were performed at the ID31 high-energy buried interface beamline at ESRF. The white beam was pre-focused to $0.5 \mathrm{~mm} \times 0.5 \mathrm{~mm}$, monochromatised to $E_{p h}=70 \mathrm{keV}$ with a multilayer mirror, and then further focused with compound refractive lenses made from $\mathrm{Al}(r$ $=50 \mu \mathrm{m})$, resulting in a beam size of $10 \mu \mathrm{m} \times 20 \mu \mathrm{m}(v \times h$ at the sample position). Images were recorded using a Pilatus $3 \mathrm{X} 2 \mathrm{M}$ CdTe detector ( $1475 \times 1679$ pixels) placed at $0.7 \mathrm{~m}$ from the sample.

The experiments were performed in a custom-designed thin layer flow cell for in situ TSD studies (Fig. 2), made from PEEK and FFKM. Electrodeposition of Bi took place in the $4 \mathrm{~mm} \times$ $2 \mathrm{~mm} \times 0.5 \mathrm{~mm}$ flow channel $(/ \times w \times h)$. The $1.5 \mathrm{~mm}$ wide inlet channel was expanded to the channel width of $2 \mathrm{~mm}$ using four equally spaced distribution channels. This arrangement was mirrored for the outflow. In addition to the sample and the flow channel, the X-ray beam also penetrated a $200 \mu \mathrm{m}$ thick PEEK window.

$\mathrm{A} \mathrm{Ag} / \mathrm{AgCl}(3.4 \mathrm{M} \mathrm{KCl}, e D a q)$ reference electrode was connected to the electrolyte $4 \mathrm{~cm}$ upstream of the thin flow channel. For the counter electrode, a Pt wire was placed $1 \mathrm{~cm}$ downstream of the channel. Potentials were controlled using a potentiostat (Ivium 
CompactStat). The cell was connected to a liquid handling system for remote-controlled electrolyte exchange. Before the experiment, the cell was cleaned by immersion in a diluted mixture of $\mathrm{H}_{2} \mathrm{SO}_{4}$ and $\mathrm{H}_{2} \mathrm{O}_{2}$.

\subsection{Samples and electrolytes}

The substrate was a $A u(100)$ single crystal (diameter $10 \mathrm{~mm}$, thickness $2 \mathrm{~mm}$ ). In the centre of the substrate, a borehole of $2 \mathrm{~mm}$ diameter was machined from the backside using a sparkerosion process, leaving only a $200 \mu \mathrm{m}$ thin $\mathrm{Au}(100)$ membrane which was penetrated with $\mathrm{X}$-rays (Surface Preparation Laboratory, orientation disorder $<0.1^{\circ}$, roughness after polishing $<0.03 \mu \mathrm{m})$. The sample was sputtered and annealed in a vacuum system and, prior to the experiment, annealed in an induction oven for $20 \mathrm{~min}$ at $650^{\circ} \mathrm{C}$. Bi was electrodeposited onto $\mathrm{Au}(100)$ from $0.1 \mathrm{M} \mathrm{HClO}_{4}$ containing $0.15 \mathrm{mM}$ or $0.5 \mathrm{mM} \mathrm{Bi}^{3+}$, made from $70 \% \mathrm{HClO}_{4}$ (Merck, Suprapur), $\mathrm{Bi}_{2} \mathrm{O}_{3}$ (Sigma-Aldrich, $99.999 \%$ trace metals basis) and ultrapure water (Elga Purelab), using a constant electrolyte exchange speed between 0 and $10 \mu \mathrm{l} / \mathrm{s}$ and a constant potential.

\subsection{Transmission X-ray microdiffraction experiments}

The geometry of the diffraction experiments is illustrated in Fig. 1. At high energies, the curvature of the Ewald sphere is low and the nearly perpendicular incidence of the X-ray beam onto the sample used in TSD means that the Ewald sphere is more or less parallel to the interface. Therefore, in this geometry, the two axes of the detector correspond to the $q_{x}$ and $q_{y}$ (in-plane) directions and diffraction spots appear wherever the Ewald sphere intersects with Bragg reflections, CTRs or adlayer rods, revealing the full in-plane symmetry of every component of a multi-layered interface. At exactly vertical incidence $\left(\theta=0^{\circ}\right)$, the out-of-plane component $q_{z}$ is zero in the middle of the detector and increases with radial symmetry towards the edges. By changing $\theta$, the Ewald sphere can be rotated (around $q_{y}$ in Fig. 1), causing its intersection positions to move 'up' the CTR on one side and 'down' the CTR on the other side of the image. This way, any value of $q_{z}$ along the rod can be selected to appear on the detector. In the actual experiments, $\theta$ was chosen so that a Bi Bragg peak located at $q_{z}=$ 0 , typically the $\mathrm{Bi}(01 \overline{4})$ peak, was intersected by the Ewald sphere. Spatial maps were produced by performing, at several positions along the flow channel, scans of the X-ray beam along the direction perpendicular to the flow. In the following, we denote the latter as "horizontal" and the direction parallel to the flow as "vertical", which corresponds to the graphical representations of the maps in Fig. 5.

A $2 \mathrm{~mm} \times 2.5 \mathrm{~mm}(v \times h)$ section in the centre of the flow channel was scanned after completion of the deposition process and the introduction of Bi-free electrolyte. Scanning the $20 \mu \mathrm{m} \times 10 \mu \mathrm{m}$ beam over the sample in $11 \times 101$ steps of $5 \mathrm{~s}$ acquisition time produced a map of 1111 full diffraction images, each of them representative for a $180 \mu \mathrm{m} \times 25 \mu \mathrm{m}$ section of the channel. After stripping the Bi deposit, an identical scan was performed for the bare $\mathrm{Au}(100)$ electrode and subtracted from the first dataset. This difference imaging mode largely removes the static background and allows the identification of thin Bi coverages (down to a few $\mathrm{ML}$ ) at short acquisition times. 
To obtain the structural parameters of film and substrate from the large set of XRD images, automated fitting routines were employed. Several different Bragg peaks were selected for analysis, as described below. Rectangular cut-outs centred on each of these peaks were produced from the full diffraction image and then rotated as necessary to move the in-plane radial direction parallel to the horizontal axis of the image. This way, radial and azimuthal cuts of the peaks could be obtained by integrating over the images' columns and rows, respectively. Both profiles were fitted with a Gaussian and a linear background. The fit quality was checked by inspecting the results at selected sample positions.

\subsection{Bi diffraction geometry}

Bismuth crystallises in a non-trivial structure which can be expressed using a rhombohedral or a hexagonal unit cell with the 'obverse' orientation.[20] Specifically, the (110) rh plane of the rhombohedral cell corresponds to the $(012)_{\text {hex }}$ plane of the hexagonal cell. Only the hexagonal notation will be employed from here on forward.

The $\mathrm{Bi}(012)$ surface lattice (Fig. 3) is rectangular with $a=4.75 \AA$ parallel to the $\mathrm{Bi}[01 \overline{4}]$ direction and $b=4.55 \AA$ parallel to the $\mathrm{Bi}[2 \overline{1} 0$ ] direction. [21] It features four corner atoms and one central atom, the latter being located slightly off-centre and out of plane. This lowsymmetry geometry gives rise to a high number of possible domains and diffraction spots.

\section{Results and discussion}

\subsection{Overall Bi deposition behavior}

Figure $4 \mathrm{~A}$ presents the voltammogram of $\mathrm{Au}(100)$ in $\mathrm{Bi}^{3+}$ containing electrolyte in the TSD thin film cell. In agreement with the literature, Bi electrodeposition on $\mathrm{Au}(100)$ commences with the formation of an underpotential deposition (UPD) layer at $0.26 \mathrm{~V}$, followed by Bi bulk deposition below $-0.03 \mathrm{~V}$.[6, 22] The Bi stripping peak was used to assess the total deposition charge $Q_{d e p}$, from which the average thickness of the resulting Bi film was calculated. This was employed to determine the deposition rate for different electrolyte flow rates between $0 \mu \mathrm{l} / \mathrm{s}$ and $10 \mu \mathrm{l} / \mathrm{s}$. Figure $4 \mathrm{~B}$ shows the deposition rate (in $\mathrm{ML} / \mathrm{s}$ ) averaged over a deposition time of $t_{\text {dep }}=60 \mathrm{~s}$ at $-0.13 \mathrm{~V}$ in $0.1 \mathrm{M} \mathrm{HClO}_{4}+0.5 \mathrm{mM} \mathrm{Bi}^{3+}$. Deposition from stagnant solution yielded a very low deposition rate of $0.06 \mathrm{ML} / \mathrm{s}$ and a total of $\approx 4 \mathrm{ML}$. Considering that the flow channel volume is $4 \mathrm{~mm}^{3}$, the maximum amount of $B i$ that can be deposited from a stagnant solution is $\approx 16 \mathrm{ML}$. From $1 \mu \mathrm{l} / \mathrm{s}(0.35 \mathrm{ML} / \mathrm{s})$ to $10 \mu \mathrm{l} / \mathrm{s}(0.98 \mathrm{ML} / \mathrm{s})$, a roughly linear correlation was observed (Fig. 4B). Under these conditions, the amount of Bi consumed from the electrolyte is negligible (between 3 and $10 \%$ ) and the main effect of the flow rate is to increase convection and reduce the diffusion layer thickness. For this regime of high electrolyte velocity $v$, a $v^{1 / 3}$ scaling relationship for the average transport rate to the electrode surface has been derived by Kjeang et al.[23] Applying the quantitative relationship of that work to our experimental conditions and assuming a $\mathrm{Bi}^{3+}$ diffusion constant of $\mathrm{D}=1.3 \cdot 10^{-5} \mathrm{~cm}^{2} / \mathrm{s}[24]$, reasonable agreement to our measurements is obtained (Fig. 4B, line), although the dependence on the flow rate seems to deviate from a $v^{1 / 3}$ relationship. The quantitative

agreement of the deposition rates with the theory by Kjeang et al. may be somewhat fortuitous, taken into account that substantial entrance effects are to be expected in our cell, which lead to rather non-uniform flow in our system (see below). 
To determine the Bi orientation and epitaxy, Bi was deposited on $\mathrm{Au}(100)$ for $20 \mathrm{~s}$ at $E=-0.08$ $V$ at a flow rate of $2 \mu \mathrm{l} / \mathrm{s}$. The average thickness of the resulting film was electrochemically determined as 4.5 ML. The TSD image $\left(\theta=0^{\circ}\right)$ after the deposition (Fig. 4C, D) confirms a (012) surface normal direction. In particular, a prominent set of strong Bragg peaks with $90^{\circ}$ symmetry can be observed (black and blue labels along the $A u[100]$ and [010] main directions in Fig. 4D). They correspond to the $(01 \overline{4})$ and $(2 \overline{1} 0)$ reflections of $\mathrm{Bi}(012)$. Indexing the remaining reflections (red, green, magenta and purple markings in Fig. 4D) shows that there are four additional domains oriented at a rotation angle $\alpha= \pm 28^{\circ}$ relative to the Au main directions. This results in a total of six different $\mathrm{Bi}$ domains and gives the diffraction image a twelve-fold symmetry. The intensity of the $\pm 28^{\circ}$ rotated domains is only $\approx 10 \%$ of the domains that are aligned with the $\mathrm{Au}[100]$ directions $\left(\alpha=0^{\circ}\right)$ when measured at the respective $L=0$ Bragg position for each of the peaks. We assign the lower intensity of rotated domains to a lower nucleation probability for their formation rather than a smaller domain size for the following reasons: Firstly, the lateral domain sizes of rotated and aligned domains are very similar (see below). Secondly, the vertical growth of the Bi crystallites is not expected to depend significantly on their in-plane orientation, whereas their nucleation on the $\mathrm{Au}(100)$ surface may well be affected by the epitaxial relationship. This domain arrangement is constant in different deposit regions and is in agreement with previous observations.[25] Zheng also showed the presence of additional, weakly oriented domains at $\pm 15^{\circ}$ and $45^{\circ}$ domains for $\mathrm{Bi}(012)$ deposits of $20 \mathrm{ML}$ and below, which vanish for thicker films. Neither of these additional domains is observed in our experiments. However, it is possible that the diffraction from these weak and less ordered domains is below the detection limit for TSD when short acquisition times are used. Matsushima et al. reported that the initial stages of $\mathrm{Bi}$ ultrathin film deposition on $\mathrm{Au}(100)$ show (001)-oriented epitaxial growth before transitioning into the (012) orientation.[26] We did not observe any orientation other than (012) and the study of such ultrathin films is out of scope for our study.

\subsection{Local Bi deposition behavior}

We now focus on thicker films to study the spatial variation of the deposit. For this purpose, we deposited $27 \mathrm{ML}$ (averaged over the whole sample) at $E=-0.13 \mathrm{~V}$ from $0.1 \mathrm{M} \mathrm{HClO}_{4}+0.15$ $\mathrm{mM} \mathrm{Bi}^{3+}$ at a flow rate of $10 \mu \mathrm{l} / \mathrm{s},\left(t_{d e p}=100 \mathrm{~s}\right)$. Again, this thickness is in good agreement with the value calculated for transport-limited deposition at high electrolyte velocity from the expression given by Kjeang et al.[23] The formed deposit was scanned in the sample center, where the Au crystal is only $200 \mu \mathrm{m}$ thick, with a $\mu \mathrm{m}$-sized X-ray beam. From the TSD data, 2D maps of the in-plane structural parameters can be generated by fitting intensity, position, as well as radial and azimuthal widths of the Bi diffraction peaks (Fig. 5). Because a full image of the in-plane reciprocal space exists for each sample position, such maps can be created from each of the many different Bi diffraction peaks visible in Fig. 4. This allows identifying differences between $\mathrm{Bi}$ grains with different orientations, which will be explored later. To optimize the acquisition conditions and reduce the acquisition time, we set $\theta$ at a value allowing to maximize the diffracted intensity of the two chosen Bi reflections.

The $\mathrm{Bi}(01 \overline{4})$ peak was chosen for quantitative determination of the deposit structure since the other two intense reflections, $(\overline{1} 1 \overline{2})$ and $(10 \overline{2})$, overlap. Two of the six rotational domains 
of the Bi film were analyzed: The domain oriented at $\alpha=0^{\circ}$ (Fig. 5A-D), i.e. along the Au[100] direction, which is the dominant orientation, and one of the weaker domains that are $\alpha=$ $\pm 28^{\circ}$ rotated (Fig. $5 \mathrm{E}-\mathrm{H}$ ). A tilt angle of $\theta=2.1^{\circ}$ was chosen together with a suitable sample rotation around the $q_{z}$ axis to ensure that the intersection between the Ewald sphere and both of the analyzed reflections is at $q_{z} \approx 0$, i.e. at the Bragg peak centers. This enables easier correlation of the Bragg peak intensity profiles with the real space parameters of the film.[16] We also analyzed the scattering of the Au substrate at the positions of $A u(2,0) C T R$ in the TSD images at the same $\theta$ value $\left(2.1^{\circ}\right)$. This position is near the $A u(200)$ Bragg reflection and very close to the $\mathrm{Bi}(01 \overline{4})$ position. The resulting Au maps show rather uniform peak intensities and peak positions inside the borehole, except on the left side of the map where significant deviations are found (see Fig. S1 in supplementary information). These can be attributed to a local deformation of the $200 \mu \mathrm{m}$ thick Au membrane in this area, which induces a local tilt of the diffracted Au planes and, due to the strong non-linear intensity changes of the Au CTR near the Bragg peak, large deviations in the diffracted intensity. Because such tilt in the $\mathrm{Au}$ lattice may also affect the data for the Bi peak, we do not include the area where the substrate is deformed in the subsequent analysis.

We first discuss the data for the $\mathrm{Bi}(01 \overline{4})$ peak at $\alpha=0^{\circ}$, i.e., along the $\mathrm{Au}[100]$ main direction. The spatial map of the Bi film thickness, expressed in number of monolayers (Fig. 5A), can be obtained from the Bragg peak intensity in each point of the map $I(x, y)$, where $x$ and $y$ describe the coordinates on the sample. In the transmission geometry, the Ewald sphere intersects the Bragg peak only in a very narrow range of $L$. The detected intensity $I(x, y)$ therefore corresponds to the height of the Bragg peak at $L=0$, which is proportional to the square of the number of monolayers $N_{z}^{2}(x, y)$ as described in more detail in the supporting information. This allows determination of the thickness without acquiring the full peak profile along $L$. As visible in Fig. 5A, the deposit thickness is highly heterogeneous in the $2 \mathrm{~mm}$ diameter X-ray accessible area where the $A u(100)$ substrate has been thinned down to $200 \mu \mathrm{m}$ (indicated by dashed circle). In the most upstream area, the deposit can be up to $40 \mathrm{ML}$ thick, whereas near the channel side walls and further downstream the average coverage is lower than $5 \mathrm{ML}$ (including the Bi UPD layer), which is the experimental limit for reliable detection of the $\mathrm{Bi}$ Bragg peaks under the employed microdiffraction conditions. Between the location of maximum thickness and the negligible deposits $1.5 \mathrm{~mm}$ further downstream, the amount of deposited Bi decreases roughly linearly along the channel (Fig. 6B). This decay is attributed to a gradual increase in the thickness of the diffusion layer, which is itself a result of the convection gradient in the cell: Near the electrolyte inlet with its four distribution channels and the vertical step caused by the FFKM seal (see Fig. 2), the liquid flow is highly nonuniform, increasing local convection. This entrance effect is gradually reduced further downstream where a smooth laminar flow profile is established and the diffusion layer thickness thus is increased. Unfortunately, the entrance region cannot be directly monitored by TSD because of the high X-ray absorption by the single crystal substrate outside the borehole, which prevents a more detailed investigation of these effects. Nevertheless, comparison of the TSD and the electrochemical data indicates that only $\approx 4 \%$ of the total $\mathrm{Bi}$ deposit is located in the central area, accessible to the diffraction studies, supporting a strongly enhanced deposition in the $1 \mathrm{~mm}$ long region separating the channel entrance and 
the sample borehole. In the horizontal profile, the Bi film thickness is highest in the center of the channel and gradually decreases towards the sides (Fig. 6A), which is likely caused by the flow field in the cell. While this profile is expectedly symmetric between $1 \mathrm{~mm}$ and $1.5 \mathrm{~mm}$ from the electrolyte inlet, we observe a clear asymmetry in the lower part of the thickness map, with less Bi on the left hand side.

Besides the film thickness, other deposit properties, which are inaccessible by any other in situ technique, can be determined, such as strain, grain size and crystal orientation (Fig. 5BD). Such variations in the structural properties are important as they influence the mechanical and electronic properties of the deposit.[27] Calculation of these structural parameters requires fitting the width and position of the Bragg peak rather than just measuring its intensity. This is only possible if the peak is clearly above the background signal, and thus was only performed at positions where the intensity exceeded a certain signal-to-noise threshold equivalent to a local thickness of about $2.5 \mathrm{ML}$ for the $\alpha=0^{\circ}$ domain. For this reason, no values are given for the outer areas, where the Bi film is thinner than the threshold thickness (indicated by white color in maps).

The in-plane lattice strain of the deposit can be calculated from the in-plane radial position of the $\mathrm{Bi}(01 \overline{4})$ peak via $\varepsilon=1-q_{r} / q_{r}$, bulk (positive values refer to tensile strain, i.e., an increased real space lattice constant; the value of $q_{r}$, bulk for bulk Bi was obtained from the Bi crystals with $>30 \mathrm{ML}$ thickness). Fig. 5B shows that the strain distribution in the Bi film is rather heterogeneous. In the region closest to the electrolyte inlet, where the Bi deposit is thick, the strain is negligible. Between $1.6 \mathrm{~mm}$ and $2.0 \mathrm{~mm}$ downstream from the inlet, the strain is very heterogeneous, with pronounced local changes between almost strain-free and high tensile strain areas. Along the direction perpendicular to the channel, where the spatial resolution is much higher, we observe abrupt strain changes correlated with changes in the local $\mathrm{Bi}$ thickness (Fig. 6C-E). Even further downstream, from $2.2 \mathrm{~mm}$ to the edge of the deposit, we observe a notable compressive strain of $-0.2 \%$ to $-0.6 \%$.

The in-plane radial width of the Bragg peak is determined by the in-plane grain size of the Bi film and the in-plane variation of the strain within the area probed by the X-ray beam. These two quantities can be separated by measuring several Bragg peaks with different $q$ values.[28] For the electrodeposited $\mathrm{Bi}$ film on $\mathrm{Au}(100)$, no dependence of the radial width on the in-

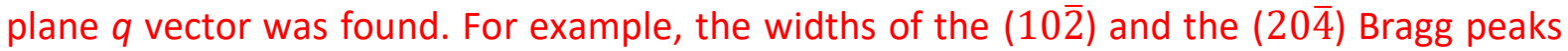
averaged over the symmetrically equivalent reflections were $0.041 \pm 0.004$ and $0.042 \pm 0.004$ $\AA^{-1}$, respectively. These peaks are the innermost $\left(q_{r}=1.9139 \AA^{-1}\right)$ and outermost $\left(q_{r}=\right.$ $3.8278 \AA^{-1}$ ) in-plane Bi reflections which are accessible in our experiment. The absence of any dependence of the in-plane radial width on the in-plane $q$ vector shows that the influence of strain variation on peak width is negligible and that the in-plane grain size of the Bi film can be determined from the in-plane radial width of the Bragg peak via $2 \pi / \sigma_{F W H M}$, rad. [29] The obtained map shows a clear correlation of the grain size with the film thickness and exhibits a value of $38 \mathrm{~nm}$ for the thickest deposits (Fig. 5C).

The azimuthal broadening $\sigma_{F W H M}, a z$ of the $\mathrm{Bi}(01 \overline{4})$ peak is comprised of two components: a contribution $\sigma_{\text {grain }}$ originating from the previously determined grain size and a second contribution $\sigma_{\text {rot }}$ originating from the orientation disorder of the different domains. Assuming 
isotropic grain size at each map position, the rotational disorder $\sigma_{\text {rot }}$ can thus be extracted from $\sigma_{F W H M} a z^{2}=\sigma_{\text {grain }}{ }^{2}+\sigma_{\text {rot }}{ }^{2}$ (Fig. 5D). Most parts of the Bi deposit exhibit a rotational disorder of $1.5^{\circ}$ (FWHM). A portion of the film shows increased disordering of up to $\approx 2^{\circ}$, mostly on the right side of the map.

Analogous maps of the structural parameters were obtained from the $\mathrm{Bi}(01 \overline{4}) \mathrm{Bragg}$ reflections belonging to the $+28^{\circ}$ rotated domains. Because the surface fraction covered by these domains is much smaller than that covered by the aligned domains, the intensity of the Bragg peaks is only sufficient for quantitative peak fitting for local thicknesses $\geq 8 \mathrm{ML}$. Apart from the poorer statistics, the obtained maps (Fig. 5E-H) strongly resemble those obtained for the aligned domains. The intensity map (Fig. 5E) is identical to that of the aligned domains. This supports our assumptions that the vertical growth of the crystallites is independent of the domain orientation and that the coverage ratio of the different rotational domains does not depend on the location on the sample, i.e., on the growth conditions. Also the maps of the strain (Fig. 5F) and the lateral grain size (Fig. 5G) are very similar, both with respect to the spatial distribution and the absolute values. An interesting difference between the two domains can be observed for the map of rotational disorder (Fig. $5 \mathrm{H}$ ), which is much higher for the domain oriented at $28^{\circ}$ (up to $3^{\circ}$ ). This behaviour is not unusual for epitaxial films that are rotated away from the main lattice directions, as the orientation here is fixed by the coincidence conditions between substrate and adlattice, which vary gradually.

The data presented in Fig. 5 provide clear evidence for correlations between the different structural parameters, especially for correlations with the film thickness. To analyze these correlations in more detail, we plot in Fig. 7 the strain, grain size, and rotational disorder against the local thickness for the $\mathrm{Bi}(01 \overline{4})$ Bragg reflections with $\alpha=0^{\circ}$ (i.e., those oriented along the $\mathrm{Au}[100]$ main direction). Similar structural trends were also found for $28^{\circ}$ rotated domains (see supporting information, Fig. S2). These plots effectively correspond to an ensemble of several hundred local deposits grown under different deposition rates induced by different local transport conditions. To highlight trends, the results from different sample areas with characteristic structural behavior are color-coded. We exclude the leftmost part of the map $(\leq-0.5 \mathrm{~mm})$ from this analysis, because here the Bi local thickness might be underestimated due to substrate bending (see above). Furthermore, the large scatter in the data at very small thickness $(\leq 5 \mathrm{ML}$ ) likely results from the very low intensities of the corresponding Bragg peaks, leading to larger errors in the peak fitting.

These correlation plots show clear trends of the structural evolution. The local strain exhibits a rather complex dependence on film thickness (Fig. 7A). In most sample areas we find at low thickness compressive strain ( $\approx-0.4 \%)$, which increases and saturates at zero near an average thickness of $15 \mathrm{ML}$. This trend is indicated by the black line in Fig. 7A, which shows the average of points recorded in the channel center (corresponding area indicated by orange in the inset in Fig.7A). In addition, clearly larger, significantly tensile strain ( $\approx+0.25 \%)$ is found in a region near the center of the observed area (marked in red), where also the film thickness is locally larger. In this specific region of the sample, the growth conditions seem different, yielding $\mathrm{Bi}$ grains with larger strain than that in the rest of the deposit and with no clear thickness dependence. The origin of this higher local strain is unknown. 
The average lateral size of the Bi grains (Fig. 7B) exhibits an increase with average thickness, reflecting the growth of the grains. The scattering of the grain size at a given thickness appears to decrease for thicker deposits, suggesting that the grain distribution becomes more homogeneous with increasing film thickness. Inspecting the data along vertical or horizontal cuts of the map and comparing left and right parts of the analyzed region of the map shows the same trend of lateral grain size increasing with thickness.

The rotational disorder (Fig. 7C) has a spatially dependent trend. In the region with high tensile strain (red symbols) we observe that the rotational disorder decreases approximately linearly from a value $>2^{\circ}$ down to $\approx 1.25^{\circ}$ with increasing thickness. Although the same trend is discernible in the remaining areas in the channel center (orange points), it is much less pronounced, leading on the average (black line) to a decrease from $\approx 1.5^{\circ}$ to $\approx 1.2^{\circ}$ between 5 and $35 \mathrm{ML}$ thickness. According to a more detailed correlation analysis, the rotational disorder tends to decrease with increasing thickness and grain size, parallel to the relaxation in tensile or compressive strain. These structural changes with increasing film growth may be caused by relaxation processes at the deposit's grain boundaries, which determine both strain and small-angle misorientation of the grains.

The origin of the observed differences between different areas in the correlation analysis is currently not clear. Nevertheless, the different trends show that structural parameters may have unexpected significant spatial dependence, which can only be revealed using a structurally and spatially sensitive technique such as TSD. Accessing this information is important to understand the growth behavior in microfluidic conditions, but would be impossible for macroscopic techniques.

\section{Conclusions}

The example given in this work, Bi electrodeposition on $\mathrm{Au}(100)$, demonstrates the capabilities of high-energy X-ray scattering in transmission geometry for detailed, spatially resolved in situ characterization of electrodeposited thin films and the underlying substrate surfaces in microfluidic channels. We have shown that a range of structural parameters, such as local deposit thickness, strain, and grain orientation, can be obtained simultaneously, allowing mapping of the deposit with a spatial resolution of a few micrometers. The latter is only possible by the TSD method, where the resolution is given by the small diameter of the focused X-ray beam. Although a variety of diffraction- or imaging-based techniques is capable of probing electrodeposited films on the $\mathrm{nm}$ or atomic scale, such as AFM, STM, SEM, LEED or EDX, with some of them also enabling in situ studies, TSD offers unique advantages. As shown by our studies, it allows combining precise local characterization of crystal structure and texture with in situ studies and the capability to probe buried interfaces, making it a valuable new tool for electrochemical materials processing and related areas. In particularly, it excellently complements conventional grazing incidence surface diffraction. The latter allows to measure the average of structural parameters with high sensitivity and precision, whereas TSD adds localized information. The electrodeposit used in this work is rather stable in air and spatially resolved characterizations could in principle also be acquired by ex situ

methods. However, this option is not available for many other systems. Especially very thin films may easily change (e.g. oxidize) during sample transfer. In addition, the TSD method is 
not restricted to studies of electrodeposition, but should be also useful for studies of other electrochemical or wet-chemical processes, e.g., for determining structural changes in battery model systems.

In the admittedly rather simple proof-of-principle experiments presented here, we employed transport-limited electrodeposition conditions, which allowed us to obtain a wide variation of deposits grown simultaneously at different deposition rates. As illustrated, this enables cross-correlation of the structural parameters of the local deposits, which may provide interesting insights into the growth process such as the unexpected heterogeneity of the structural parameters as a function of thickness. By further experimental improvements, in particular well-defined microfluidic cells with quantitatively determined hydrodynamic conditions, this approach may become a powerful tool for high-throughput screening in electrochemical material synthesis. For example, laminar flow cells with two inlets may allow to introduce and mix two different electrolytes in the cell, which could be used to produce compositional gradients. Vice versa, the high spatial resolution of TSD will allow to characterize the deposits formed in such cells and thus provides a powerful analytic tool to probe the manufacturing process. With the field of microfluidics steadily turning towards $3 \mathrm{D}$ printing compared to lithographic methods, [30] such devices will be increasingly faster and easier to produce and evaluate. In combination, microfluidic cells and synchrotron-based TSD therefore may offer interesting new opportunities for electrochemical material science.

\section{Acknowledgements}

The authors gratefully acknowledge financial support by the Deutsche Forschungsgemeinschaft and the Agence Nationale de la Recherche (ANR) via project EC-MEC (MA 1618/2020 and ANR-15-CE30-0024-01) and thank the ID 31 staff for technical support.

\section{References}

[1] I. K. Robinson, Acta Cryst., A54, 772 (1998)

[2] E. Vlieg, in Surface and Interface Science, Vol. 1, K. Wandelt, Editor, pp. 375-425, WileyVCH, New York (2014)

[3] Z. Nagy and H. You, Electrochim. Acta, 47, 3037 (2002)

[4] Y. Gründer and C. A. Lucas, Curr. Opin. Electrochem., 19, 168 (2020)

[5] J. Wang and B. M. Ocko, Phys. Rev. B, 46, 10321 (1992)

[6] S. H. Zheng, K. Krug, F. Golks, D. Kaminski, S. Morin and O. M. Magnussen, J. Electroanal. Chem., 649, 189 (2010)

[7] O. M. Magnussen, B. M. Ocko, R. R. Adzic and J. X. Wang, Phys. Rev. B, 51, 5510 (1995)

[8] M. F. Toney, J. G. Gordon, M. G. Samant, G. L. Borges, O. R. Melroy, D. Yee and L. B. Sorensen, J. Phys. Chem., 99, 4733 (1995)

[9] F. Maroun, F. Reikowski, N. Di, T. Wiegmann, J. Stettner, O. M. Magnussen and P. Allongue, J. Electroanal. Chem., 819, 322 (2018)

[10] I. M. Tidswell, N. Markovic and P. N. Ross, Phys. Rev. Lett., 71, 1601 (1993)

[11] B. M. Ocko, O. M. Magnussen, J. X. Wang and R. R. Adzic, Physica B, 221, 238 (1996)

[12] H. Keller, M. Saracino, H. M. T. Nguyen, T. M. T. Huynh and P. Broekmann, J. Phys. Chem. C, 116, 11068 (2012) 
[13] M. Nakamura, Y. Nakajima, N. Sato, N. Hoshi and O. Sakata, Phys. Rev. B, 84, 165433 (2011)

[14] T. Kondo, T. Masuda, N. Aoki and K. Uosaki, J. Phys. Chem. C, 120, 16118 (2016)

[15] J. Gustafson, M. Shipilin, C. Zhang, A. Stierle, U. Hejral, U. Ruett, O. Gutowski, P.-A. Carlsson, M. Skoglundh and E. Lundgren, Science, 343, 758 (2014)

[16] F. Reikowski, T. Wiegmann, J. Stettner, J. Drnec, V. Honkimäki, F. Maroun, P. Allongue and O. M. Magnussen, J. Phys. Chem. Lett., 8, 1067 (2017)

[17] A. Wlasenko, F. Soltani, D. Zakopcan, D. Sinton and G. M. Steeves, Phys. Rev. E, 81, 021601 (2010)

[18] J. W. Gallaway, D. Desai, A. Gaikwad, C. Corredor, S. Banerjee and D. Steingart, J. Electrochem. Soc., 157, A1279 (2010)

[19] A. Bîrzu, J. Coleman and I. Z. Kiss, J. Electroanal. Chem., 726, 27 (2014)

[20] H. Arnold, in International Tables for Crystallography, Vol. A, 5th ed., T. Hahn, Editor, p. 78, Springer, Dordrecht (2005)

[21] C. A. Jeffrey, S. H. Zheng, E. Bohannan, D. A. Harrington and S. Morin, Surf. Sci., 600, 95 (2006)

[22] M. Hara, Y. Nagahara, S. Yoshimoto, J. Inukai and K. Itaya, J. Electrochem. Soc., 151, E92 (2004)

[23] E. Kjeang, B. Roesch, J. McKechnie, D. A. Harrington, N. Djilali and D. Sinton, Microfluid. Nanofluid., 3, 403 (2007)

[24] Although the $\mathrm{Bi}^{3+}$ diffusion coefficient in the electrolyte is not known, typical values for ions in aqueous solution are of the order $10^{-5} \mathrm{~cm}^{2} / \mathrm{s}$ at room temperature. $D=1.3 \cdot 10^{-5} \mathrm{~cm}^{2} / \mathrm{s}$ corresponds to the value that provides the best match with the experimental data.

[25] S. H. Zheng, PhD thesis, York University (2011)

[26] H. Matsushima, S.-W. Lin, S. Morin and O. M. Magnussen, Faraday Discuss., 193, 171 (2016)

[27] G. R. Stafford and U. Bertocci, J. Phys. Chem. C, 113, 13249 (2009)

[28] A. S. Kurlov and A. I. Gusev, Glass Phys. Chem., 33, 276 (2007)

[29] B. E. Warren, X-Ray Diffraction, p. 253, Dover Publications, New York (1990)

[30] C. M. B. Ho, S. H. Ng, K. H. H. Li and Y.-J. Yoon, Lab Chip, 15, 3627 (2015) 


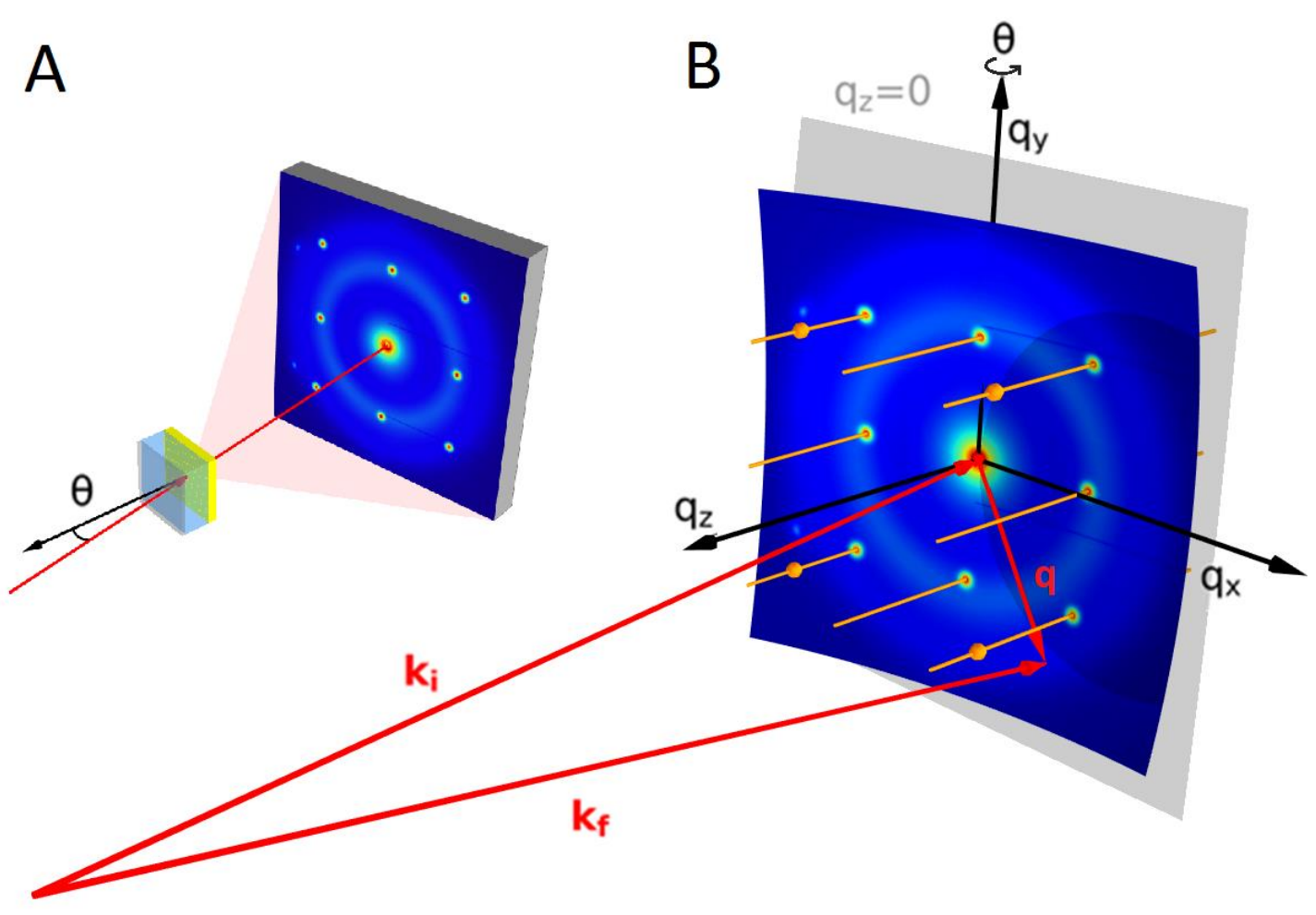

Fig. 1: Real (A) and reciprocal (B) space geometry of the TSD measurements. The X-ray beam (red) passes through electrolyte and sample at nearly perpendicular incidence. At $E_{P h}=$ $70 \mathrm{keV}$, the Ewald sphere is almost parallel to the plane spanned by the in-plane vectors $q_{x}$ and $q_{y}$, with diffraction peaks appearing on the detector at intersections with CTRs (orange). By adjusting $\theta$, the Ewald sphere can be turned around $q_{y}$, changing the $q_{z}$ value of the peaks visible on the detector. Adapted with permission from [16]. Copyright 2017 American Chemical Society. 

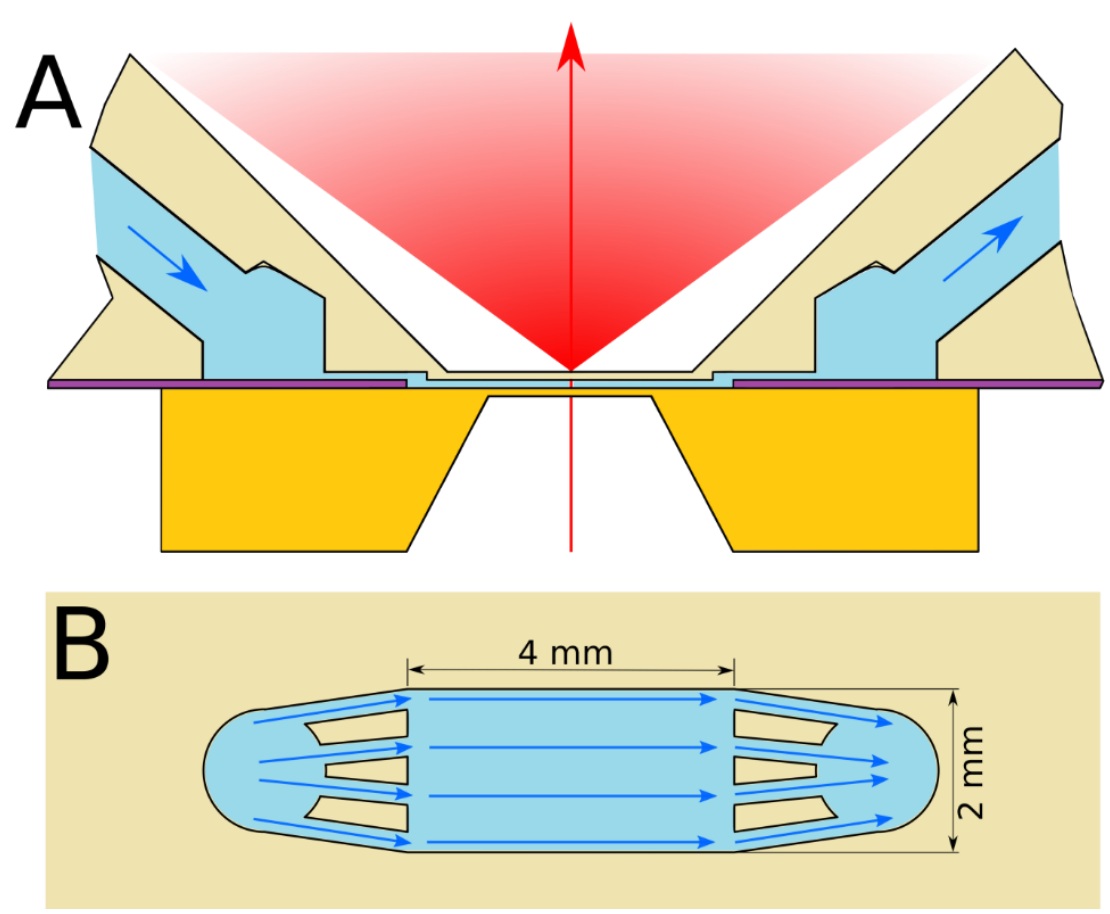

Fig. 2: (A) Cross-section of the electrochemical thin film flow cell used for in situ TSD, showing from top to bottom: (1) PEEK cell body (brown), with electrolyte in- / outlet in the top left and top right. Connections for reference and counter electrode not shown. (2) FFKM foil (purple) with rectangular cut-out for flow channel. (3) $\mathrm{Au}(100)$ single crystal (yellow) with sparkeroded borehole. The path of the X-ray beam is indicated in red. (B) Top-down view on the flow channel. The in-/ outlets and the 2.4 distribution channels (each of $0.25 \mathrm{~mm}$ width, $1.5 \mathrm{~mm}$ length and $0.1 \mathrm{~mm}$ depth) are covered by the FFKM foil, leaving only the $2 \mathrm{~mm} \times 4$ $\mathrm{mm}$ centre part exposed to the sample resting on top. 

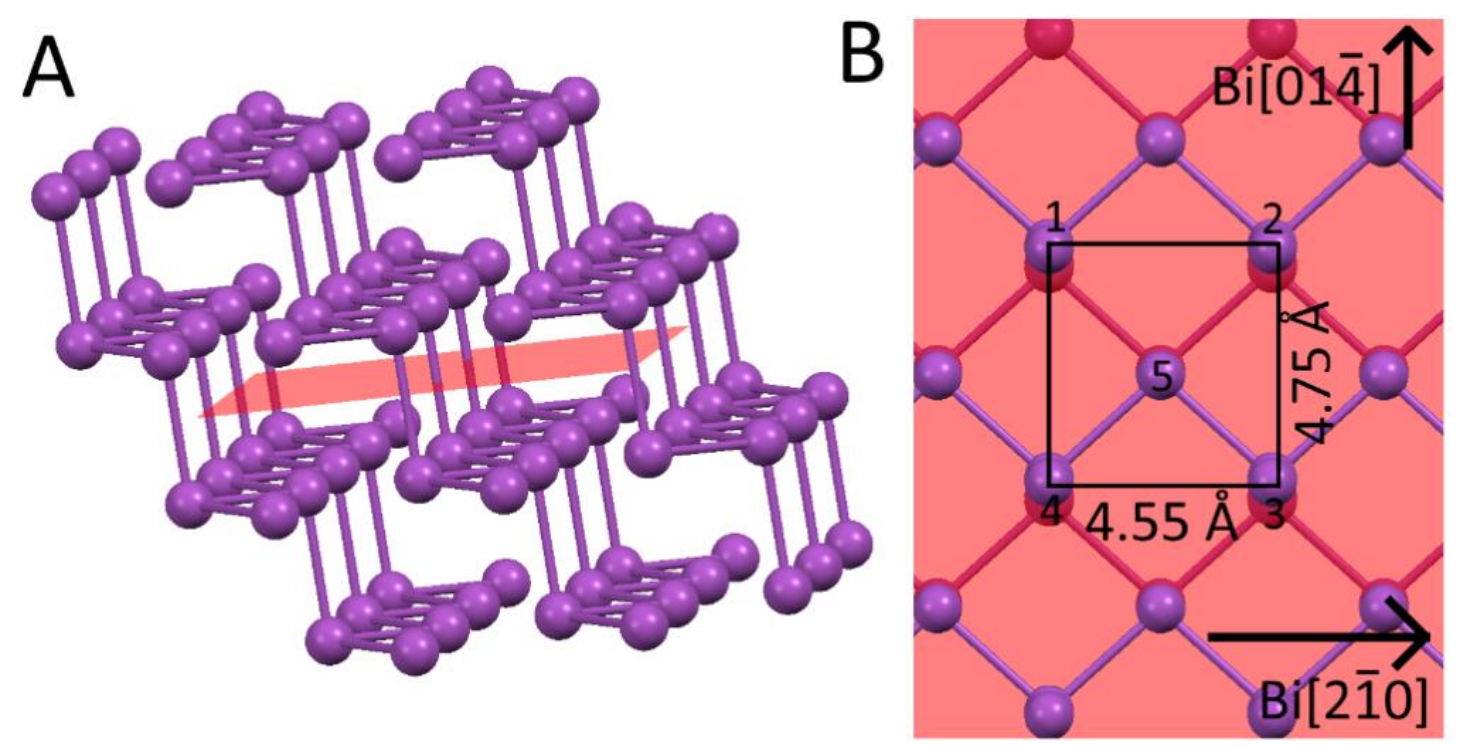

Fig. 3: (A) Real space geometry of the bulk $\mathrm{Bi}$ crystal structure. Bonds between $\mathrm{Bi}$ atoms with covalent character and a length of $3.07 \AA$ are indicated by lines. The (012) plane is shown in red. (B) Perpendicular view onto the $\mathrm{Bi}(012)$ plane with dimensions and orientation of the rectangular surface unit cell. Atoms above the plane are drawn in purple, atoms below in crimson. 

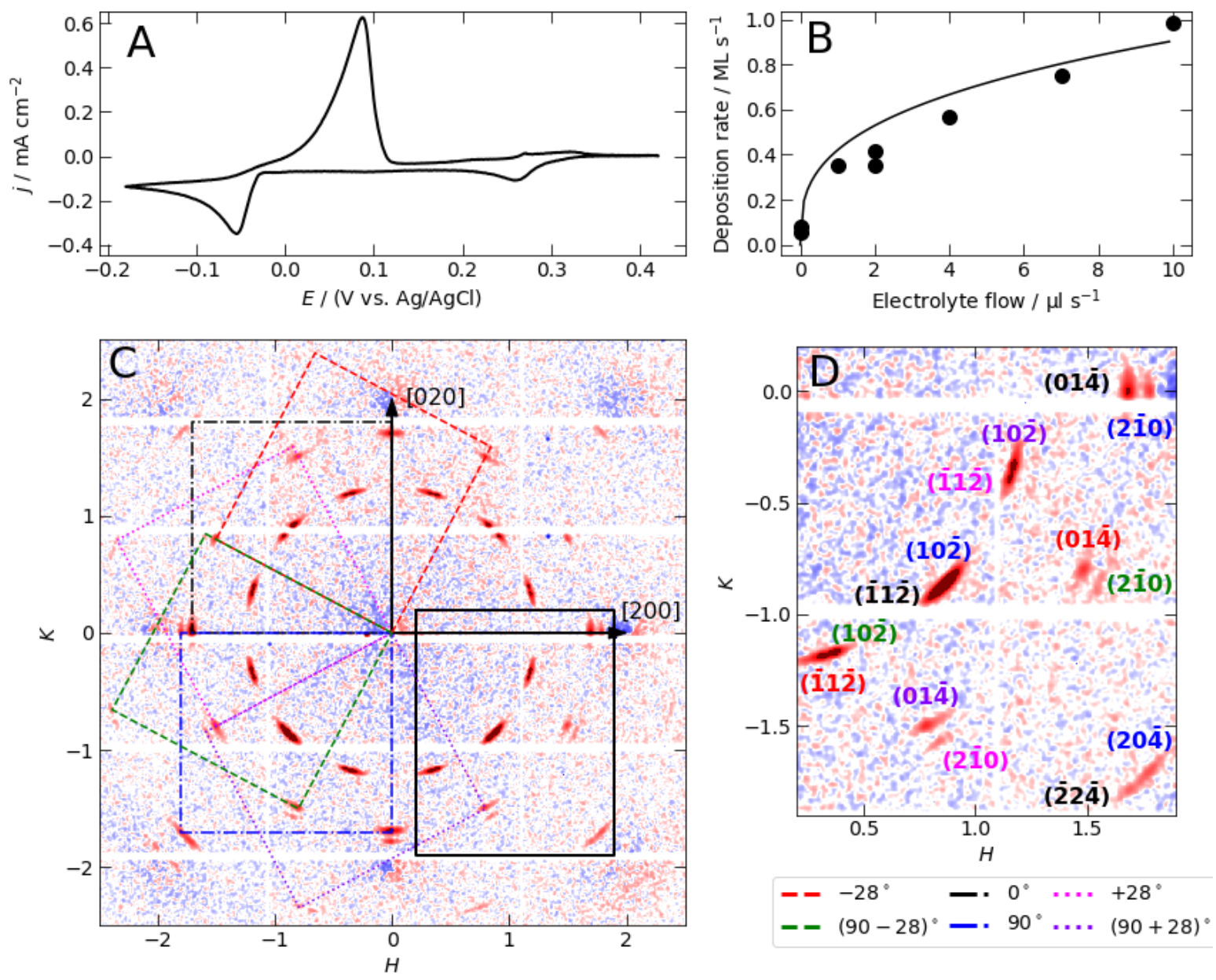

Fig. 4: (A) Cyclic voltammetry of Bi electrodeposition on $\mathrm{Au}(100)$ in $0.1 \mathrm{M} \mathrm{HClO}_{4}+0.5 \mathrm{mM}$ $\mathrm{Bi}^{3+}$, obtained at a scan rate of $10 \mathrm{mV} / \mathrm{s}$. (B) Bi deposition rate (averaged over the full sample) as a function of the electrolyte flow during deposition. All depositions were performed at $-0.13 \mathrm{~V}$ for $60 \mathrm{~s}$. (C) TSD difference image $(\theta=0)$ of a $\mathrm{Bi}(012)$ film of average thickness 4.5 $\mathrm{ML}$ electrodeposited on $\mathrm{Au}(100)$ at $-0.08 \mathrm{~V}$ and a flow rate of $2 \mu \mathrm{l} / \mathrm{s}$. The image was produced by subtracting detector images acquired for $30 \mathrm{~s}$ after and before the deposition process and applying a Gaussian blur filter for noise reduction. Red pixels signify an increase, blue pixels a decrease in intensity after deposition. Coordinates $(H, K)$ are given with respect to the $\mathrm{Au}(001)$ substrate. The $\mathrm{Au}[200]$ and $\mathrm{Au}[020]$ directions are marked by arrows. For each domain, one rectangular unit cell of the in-plane lattice in reciprocal space of the $\mathrm{Bi}(012)$ deposit is marked by dashed / dotted lines. For the $0^{\circ}$ domain (black dash-dotted unit cell), the $\mathrm{Bi}[01 \overline{4}]$ direction is parallel to $\mathrm{Au}[100]$, i.e. the horizontal axis. (D) Enlarged view of the area in the thick rectangle in $(\mathrm{C})$, in which the $\mathrm{Bi}$ peaks are indexed with respect to the hexagonal Bi bulk unit cell. 


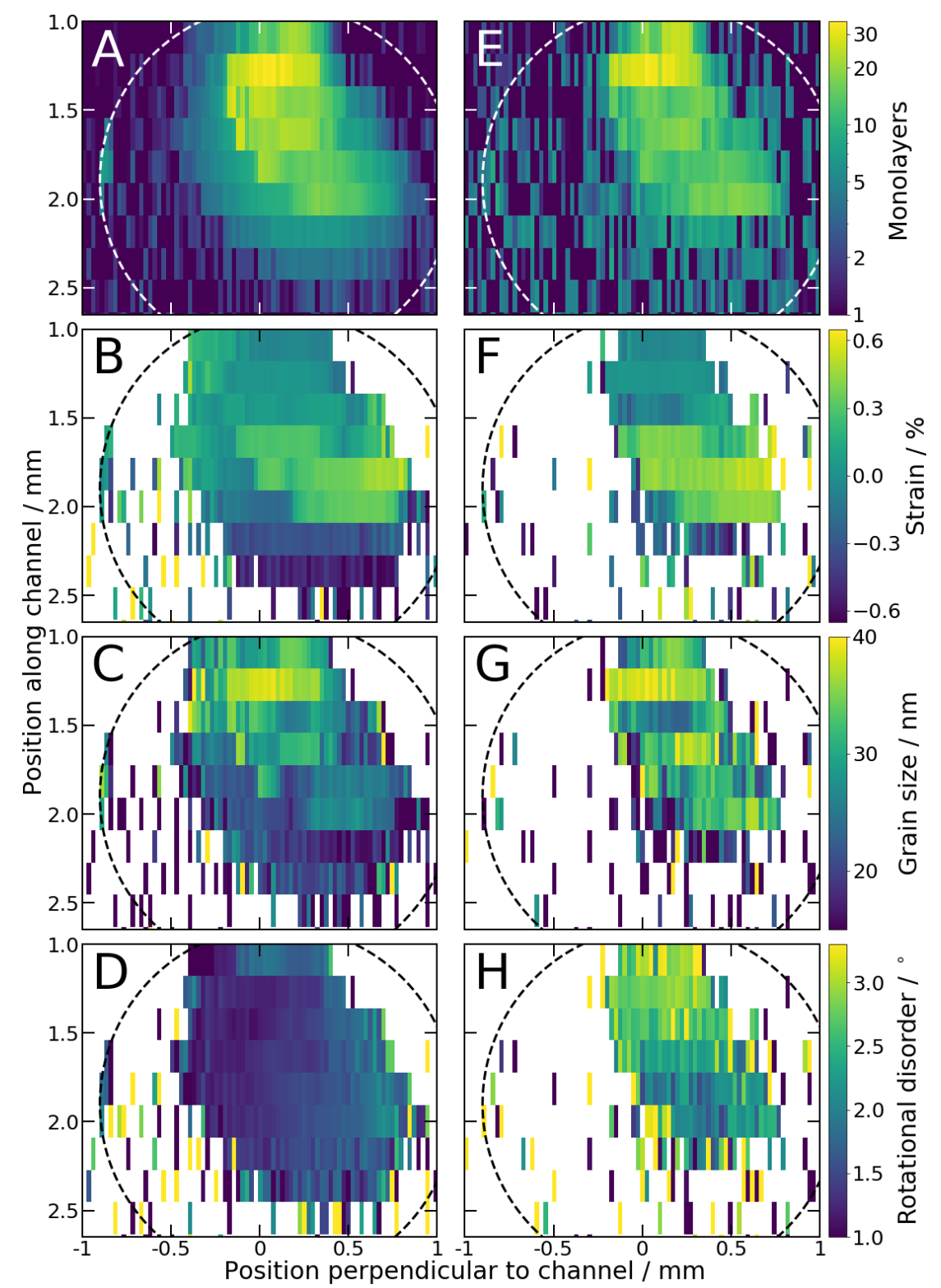

Fig. 5: Real space maps of the structural parameters of a Bi film deposited on $A u(100)$ for 100 $\mathrm{s}$ at $-0.13 \mathrm{~V}$ from $0.1 \mathrm{M} \mathrm{HClO}_{4}+0.15 \mathrm{mM} \mathrm{Bi}^{3+}$ at a flow rate of $10 \mu \mathrm{l} / \mathrm{s}$ (average coverage of the observed area $=4 \mathrm{ML})$. The $2 \mathrm{D}$ maps were obtained from an analysis of the $\mathrm{Bi}(01 \overline{4})$ peaks of the domain oriented (A-D) along and (E-H) at $+28^{\circ}$ to the [100] direction of the $A u$ substrate. Displayed is $(\mathbf{A}, \mathbf{E})$ the Bi film thickness (given as average number of monolayers per pixel), obtained from the intensity, $(\mathbf{B}, \mathbf{F})$ the tensile lattice strain, obtained from the inplane peak position, $(\mathbf{C}, \mathbf{G})$ the in-plane grain size, obtained from the radial FWHM of the peak, and $(\mathbf{D}, \mathbf{H})$ the rotational (orientation) disorder of the $\mathrm{Bi}(012)$ film obtained from the peak's azimuthal FWHM. The data are shown as a function of position within the microfluidic channel. The electrolyte flow direction was exactly vertical from top to bottom (data starts at $1 \mathrm{~mm}$ from the electrolyte inlet due to absorption from the substrate). The full width of the channel is shown, with the left and right edges of the maps corresponding to the position of the side walls. The area of the borehole where the Au substrate is X-ray-transparent is indicated by the dashed circle. 

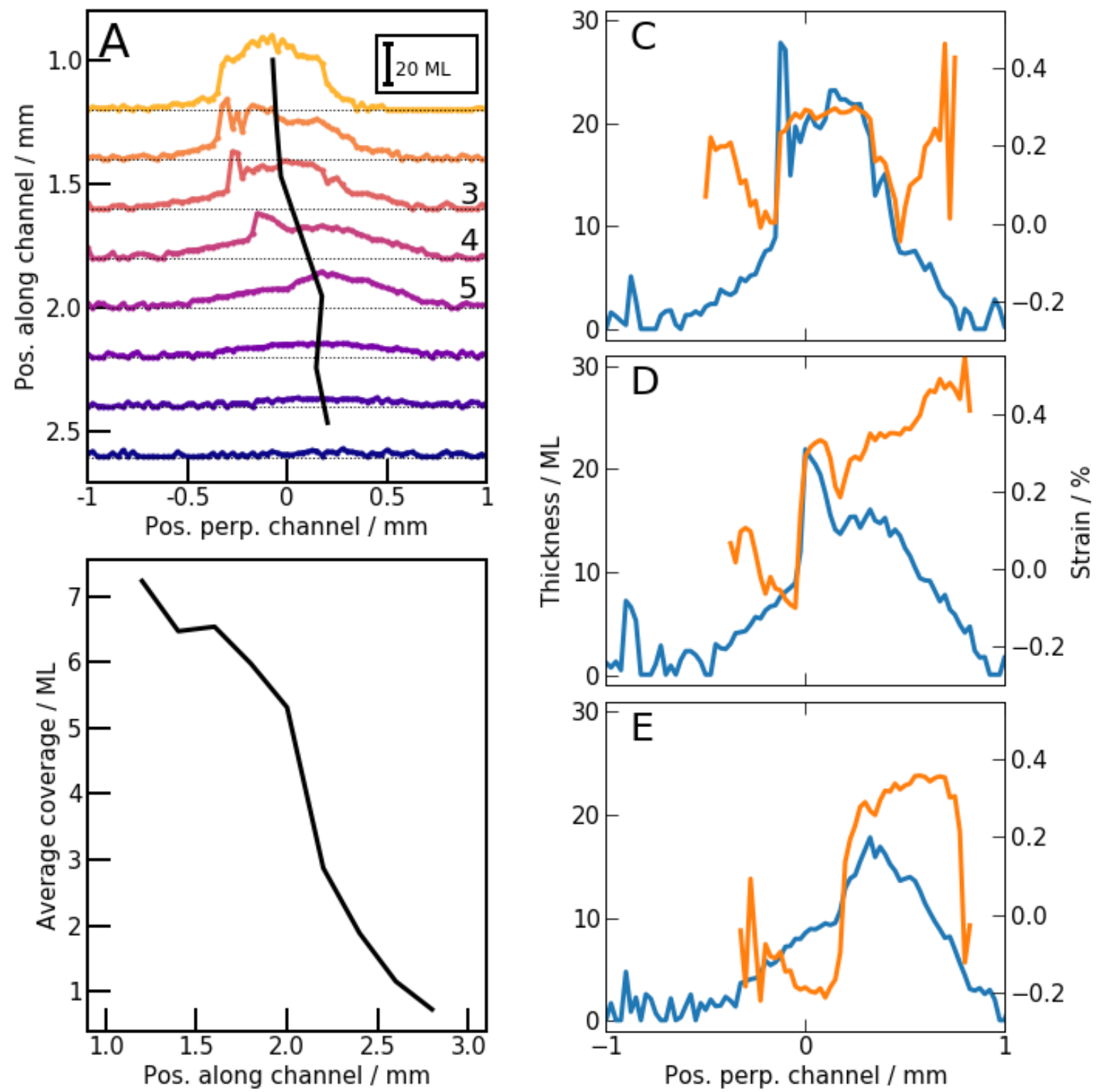

Fig. 6: (A) Data of Fig. 5A presented as stackplot, showing the film profile perpendicular to the flow direction. The centers of the profiles, obtained from a parabolic fit, are indicated by the vertical black line. (B) Average Bi amount per row in (A), obtained from integration over the respective flow-perpendicular profile. (C-E) Flow-perpendicular profiles of local film thickness (blue) and tensile strain (orange) for the $3^{\text {rd }}, 4^{\text {th }}$ and $5^{\text {th }}$ row, respectively. 

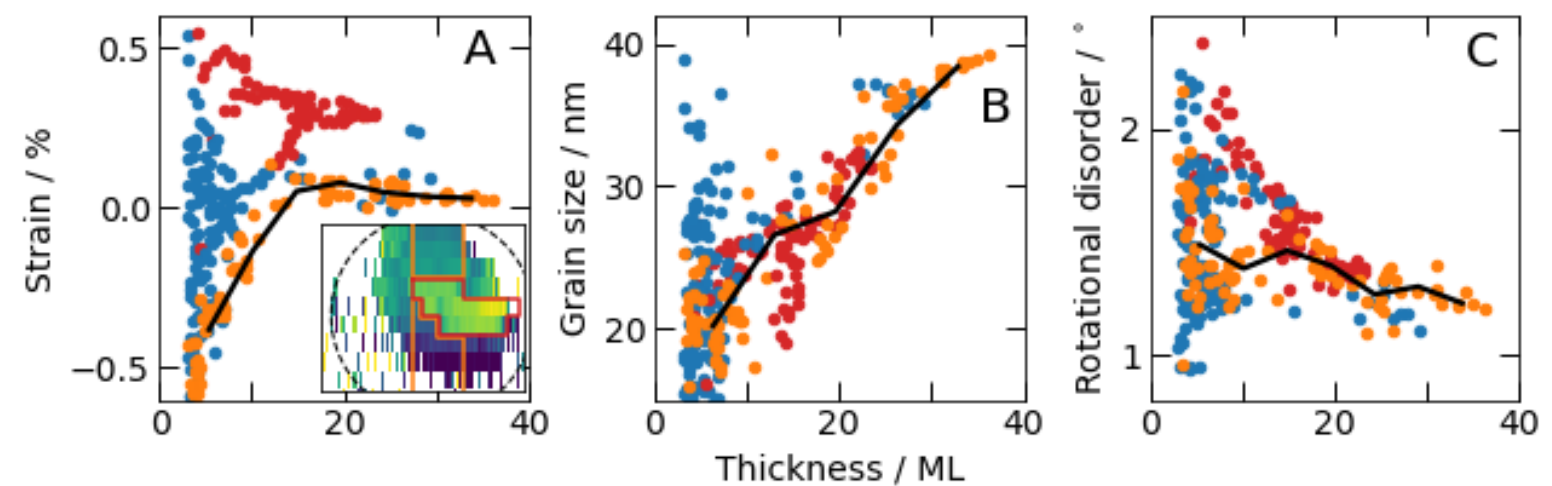

Fig. 7: Correlation scatterplots of the data in Fig. 5A-D of (A) the local tensile strain, (B) the local in-plane grain size, and (C) the local rotational disorder as a function of the local film thickness. The plots correspond to the data from the Bi domains oriented along the $\mathrm{Au[100]}$ direction. Different colors indicate data points in the central high-strain area (red), in the remaining center part of the flow channel (orange), and in the residual areas (blue), as indicated in the strain map shown in the inset of $(A)$. 